Article

\title{
New High Accuracy Analysis of a Double Set Parameter Nonconforming Element for the Clamped Kirchhoff Plate Unilaterally Constrained by an Elastic Obstacle
}

\section{Dongyang Shi and Lifang Pei *}

School of Mathematics and Statistics, Zhengzhou University, Zhengzhou 450001, China; shi_dy@zzu.edu.cn

* Correspondence: plf5801@zzu.edu.cn or plf5801@163.com

Received: 26 October 2020; Accepted: 13 November 2020; Published: 16 November 2020

Abstract: In this paper, a non- $C^{0}$ double set parameter finite element method is presented for the clamped Kirchhoff plate with an elastic unilateral obstacle. A new high accuracy error estimate with order $O\left(h^{2}\right)$ in the broken energy norm is derived by use of a series of novel approaches, including some special features of the element and an incomplete biquadratic interpolation operator. At the same time, some experimental results are provided to verify the theoretical analysis.

Keywords: elastic obstacle; Kirchhoff plate; non- $C^{0}$ FEM; double set parameter method; high accuracy error estimate

\section{Introduction}

Let $\Omega \subset \mathbf{R}^{2}$ be a bounded convex polygonal domain with a Lipschitz continuous boundary $\Gamma$. We consider a thin flat plate $\Omega \times(-d / 2, d / 2)$ clamped on its lateral boundary $\Gamma \times(-d / 2, d / 2)$, where $d>0$ is the thickness of the plate and assumed to be small. Assume that the plate is subject to a load $f \in L^{2}(\Omega), f \leq 0$ a.e. in $\Omega$ and constrained unilaterally by an elastic obstacle $\psi \in L^{2}(\Omega)$, then displacement function $u$ can be obtained by solving the weak formulation (cf. [1]):

$$
\text { Find } u \in V \text {, such that } a(u, v)+\ell(u, v)=(f, v), \forall v \in V \text {, }
$$

where $V=H_{0}^{2}(\Omega), a(w, v)=\int_{\Omega}\left[\triangle w \triangle v+(1-v)\left(2 w_{x y} v_{x y}-w_{x x} v_{y y}-w_{y y} v_{x x}\right)\right] d x d y, \ell(u, v)=$ $\int_{\Omega} \kappa(u-\psi)_{-} v d x d y, t_{-}=\min \{t, 0\},(f, v)=\int_{\Omega} f v d x d y, v \in(0,1 / 2)$ is Poisson's ratio and $\kappa \geq \kappa_{0}>0$ describes the stiffness of the obstacle.

It was shown in [1] that the displacement function $u$ can also be derived by the minimization problem:

$$
\text { Find } u \in V \text {, such that } \forall v \in V J(u) \leq J(v)
$$

with the total energy $J(v)=\frac{1}{2} a(v, v)+\frac{1}{2} \int_{\Omega} \kappa\left[(v-\psi)_{-}\right]^{2} d x d y-(f, v)$. Furthermore, the problem (2) is equivalent to the following fourth-order variational inequality of the second kind:

$$
\left\{\begin{array}{l}
\text { Find } u \in V, \text { such that } \\
a(u, v-u)+\int_{\Omega} \frac{\kappa}{2}\left[(v-\psi)_{-}\right]^{2} d x d y-\int_{\Omega} \frac{\kappa}{2}\left[(u-\psi)_{-}\right]^{2} d x d y \geq(f, v-u), \forall v \in V .
\end{array}\right.
$$


In particular, in the limit $\kappa \rightarrow \infty$, the obstacle becomes rigid, and the problem reduces to the constrained minimization:

$$
\left\{\begin{array}{l}
\text { Find } u \in K^{*}, \text { such that } \\
u=\arg \min _{v \in K^{*}}\left[\frac{1}{2} a(v, v)-(f, v)\right]
\end{array}\right.
$$

with $K^{*}=\{v \in V: v \geq \psi$ in $\Omega\}$, which is the classical displacement obstacle problem of the clamped Kirchhoff plate (cf. [2]) and is equivalent to the following fourth-order variational inequality of the first kind:

$$
\left\{\begin{array}{l}
\text { Find } u \in K^{*}, \text { such that } \\
a(u, v-u) \geq(f, v-u), \forall v \in K^{*} .
\end{array}\right.
$$

It is well known that nonconforming finite element methods (FEMs) are an attractive option for solving high order differential equations since the smoothness requirement on finite element functions is weakened. As we know, nonconforming FEMs for the problem (4), the plate obstacle problem with a rigid obstacle, have achieved fruitful results [3-9], but very few for the elastic obstacle problem (2), except [10]. Compared with the conforming FEMs studied in $[1,11], C^{0}$ continuous and discontinuous nonconforming (i.e., $C^{0}$ and non- $C^{0}$ nonconforming) FEMs were discussed in [10], and convergence analyses were established. On the other hand, the high accuracy analysis of nonconforming FEMs has been an active research area in practical computations, and many high accuracy results have been derived for variational inequalities and the boundary value problem [12-20]. However, for the plate obstacle problem (4) with a rigid obstacle, the exact solution $u$ only belongs to $H^{3}(\Omega) \cap C^{2}(\Omega)$ instead of $H_{l o c}^{4}(\Omega)$ [21,22]; thus, the lack of $H^{4}$ regularity makes it impossible to develop high accuracy analysis. Fortunately, it was shown in [10] that the solution is more regular if the obstacle is elastic. In fact, the solution of the problem (2) belongs to $H^{4}(\Omega)$ when the largest interior angles of the domain $\Omega$ are smaller than $126.283696 \ldots{ }^{\circ}$, and thus, the high accuracy analysis of FEMs for the problem (2) is possible and worth exploring.

In this paper, we attempt to present a high accuracy analysis of nonconforming double set parameter FEMs for the obstacle problem (2). The double set parameter method presented in $[23,24]$ is a useful method to construct unconventional elements, and it involves two sets of parameters. The first set is chosen to meet the convergence requirements according to the generalized patch test [25] or F-E-M-test [26], while the second set is selected to be simple so that the total number of unknowns in the resulting discrete system is small. Up to now, several nonconforming double set parameter plate elements have been successfully applied to deal with the two-sided displacement obstacle problem of the clamped plate [8], plate bending problems [16,27], the linear elasticity problem [28], the fourth-order elliptic singular perturbation problem [29-31] and so on. In this paper, a non- $C^{0}$ nonconforming double set parameter plate element is employed for the elastic obstacle problem (2). We develop a series of novel approaches including some special features of the element and an incomplete biquadratic interpolation operator so as to get the high accuracy result with order $O\left(h^{2}\right)$ in the broken energy norm. Furthermore, we carry out a numerical experiment to show the performance of the proposed method.

\section{A Twelve Parameter Double Set Parameter Element and Its Typical Properties}

In the beginning, we introduce a twelve parameter double set parameter element briefly. The readers are referred to [27] for details.

Assume that $T_{h}$ is a regular rectangular decomposition of $\Omega$. For a given $K \in T_{h}$, let its center be $\left(x_{K}, y_{K}\right)$, four vertices be $a_{i}\left(x_{i}, y_{i}\right)$, four edges be $F_{i}=\overline{a_{i} a_{i+1}}(i=1,2,3,4(\bmod 4))$, and the edge length be $2 h_{x}$ and $2 h_{y}$, respectively. Moreover, we denote $h=\max _{K \in T_{h}}\left\{h_{x}, h_{y}\right\}$. Besides, assume that $\hat{K}=[-1,1] \times[-1,1]$ is the reference element on the $\xi-\eta$ plane with a center point $(0,0)$, four 
vertices $\hat{a}_{1}=(-1,-1), \hat{a}_{2}=(1,-1), \hat{a}_{3}=(1,1)$, and $\hat{a}_{4}=(-1,1)$, and four edges $\hat{F}_{1}=\overline{\hat{a}_{1} \hat{a}_{2}}, \hat{F}_{2}=\overline{a_{2} \hat{a}_{3}}$, $\hat{F}_{3}=\overline{\hat{a}}_{3} \hat{a}_{4}$, and $\hat{F}_{4}=\overline{\hat{a}}_{4} \hat{a}_{1}$. Then, there exists an affine mapping $J: \hat{K} \longrightarrow K:$

$$
\left\{\begin{array}{l}
x=x_{K}+h_{x} \xi \\
y=y_{K}+h_{y} \eta
\end{array}\right.
$$

satisfying $J(\hat{K})=K, v(x, y)=\hat{v}(\xi, \eta)$.

The shape function space on the element $K$ is taken as:

$$
P(K)=P_{3}(K) \cup\left\{x^{4}, y^{4}\right\}=\operatorname{Span}\left\{p_{1}, \ldots, p_{12}\right\},
$$

where $p_{1}=\frac{1}{4}(1-\xi)(1-\eta), \quad p_{2}=\frac{1}{4}(1+\xi)(1-\eta), \quad p_{3}=\frac{1}{4}(1+\xi)(1+\eta), \quad p_{4}=\frac{1}{4}(1-\xi)(1+\eta)$, $p_{5}=\left(1-\xi^{2}\right), \quad p_{6}=\left(1-\eta^{2}\right), \quad p_{7}=\left(1-\xi^{2}\right) \eta, \quad p_{8}=\left(1-\eta^{2}\right) \xi, \quad p_{9}=\left(1-\xi^{2}\right) \xi, \quad p_{10}=\left(1-\eta^{2}\right) \eta$, $p_{11}=\left(1-\xi^{2}\right) \xi^{2}, \quad p_{12}=\left(1-\eta^{2}\right) \eta^{2}$, and $P_{m}(K)$ denotes the space consisting of piecewise polynomials of degree $m$ on element $K$.

The degrees of freedom are selected as:

$$
D(v)=\left(d_{1}(v), d_{2}(v), \ldots, d_{12}(v)\right)^{\prime},
$$

where:

$$
\begin{gathered}
d_{i}(v)=v\left(a_{i}\right), \quad i=1,2,3,4 \\
d_{5}(v)=\frac{1}{h} \int_{F_{1}} v d s=\int_{-1}^{1} \hat{v}(\xi,-1) d \xi, \quad d_{6}(v)=\frac{1}{h_{y}} \int_{F_{2}} v d s=\int_{-1}^{1} \hat{v}(1, \eta) d \eta, \\
d_{7}(v)=-\frac{1}{h_{x}} \int_{F_{3}} v d s=\int_{-1}^{1} \hat{v}(\xi, 1) d \xi, \quad d_{8}(v)=-\frac{1}{h_{y}} \int_{F_{4}} v d s=\int_{-1}^{1} \hat{v}(-1, \eta) d \eta, \\
d_{9}(v)=-\frac{h_{y}}{h_{x}} \int_{F_{1}} \frac{\partial v}{\partial n} d s=\int_{-1}^{1} \frac{\partial \hat{v}}{\partial \eta}(\xi,-1) d \xi, \quad d_{10}(v)=\frac{h_{x}}{h_{y}} \int_{F_{2}} \frac{\partial v}{\partial n} d s=\int_{-1}^{1} \frac{\partial \hat{v}}{\partial \xi}(1, \eta) d \eta, \\
d_{11}(v)=-\frac{h_{y}}{h_{x}} \int_{F_{3}} \frac{\partial v}{\partial n} d s=\int_{-1}^{1} \frac{\partial \hat{v}}{\partial \eta}(\xi, 1) d \xi, \quad d_{12}(v)=\frac{h_{x}}{h_{y}} \int_{F_{4}} \frac{\partial v}{\partial n} d s=\int_{-1}^{1} \frac{\partial \hat{v}}{\partial \xi}(-1, \eta) d \eta .
\end{gathered}
$$

For $v \in P(K)$, let:

$$
v=\sum_{i=1}^{12} \beta_{i} p_{i}=(P, b)
$$

where $b=\left(\beta_{1}, \beta_{2}, \cdots, \beta_{12}\right)^{\prime} \in \mathbb{R}^{12}, P=\left(p_{1}, p_{2}, \cdots, p_{12}\right)^{\prime}$.

Substituting (9) into (8), we get:

$$
D(v)=M b
$$


with:

$$
M=\left(\begin{array}{cccccccccccc}
1 & 0 & 0 & 0 & 0 & 0 & 0 & 0 & 0 & 0 & 0 & 0 \\
0 & 1 & 0 & 0 & 0 & 0 & 0 & 0 & 0 & 0 & 0 & 0 \\
0 & 0 & 1 & 0 & 0 & 0 & 0 & 0 & 0 & 0 & 0 & 0 \\
0 & 0 & 0 & 1 & 0 & 0 & 0 & 0 & 0 & 0 & 0 & 0 \\
1 & 1 & 0 & 0 & \frac{4}{3} & 0 & -\frac{4}{3} & 0 & 0 & 0 & \frac{4}{15} & 0 \\
0 & 1 & 1 & 0 & 0 & \frac{4}{3} & 0 & \frac{4}{3} & 0 & 0 & 0 & \frac{4}{15} \\
0 & 0 & 1 & 1 & \frac{4}{3} & 0 & \frac{4}{3} & 0 & 0 & 0 & \frac{4}{15} & 0 \\
1 & 0 & 0 & 1 & 0 & \frac{4}{3} & 0 & -\frac{4}{3} & 0 & 0 & 0 & \frac{4}{15} \\
-\frac{1}{2} & -\frac{1}{2} & \frac{1}{2} & \frac{1}{2} & 0 & 4 & \frac{4}{3} & 0 & 0 & -4 & 0 & 4 \\
-\frac{1}{2} & \frac{1}{2} & \frac{1}{2} & -\frac{1}{2} & -4 & 0 & 0 & \frac{4}{3} & -4 & 0 & -4 & 0 \\
-\frac{1}{2} & -\frac{1}{2} & \frac{1}{2} & \frac{1}{2} & 0 & -4 & \frac{4}{3} & 0 & 0 & -4 & 0 & -4 \\
-\frac{1}{2} & \frac{1}{2} & \frac{1}{2} & -\frac{1}{2} & 4 & 0 & 0 & \frac{4}{3} & -4 & 0 & 4 & 0
\end{array}\right) .
$$

It is easy to check that $|M|=\frac{2^{24}}{3^{4} * 5^{2}} \neq 0$; thus $b=M^{-1} D(v)$, i.e., it holds that:

$$
\forall v \in P(K), v=\left(P, M^{-1} D(v)\right) .
$$

Next, we take nodal parameters as:

$$
Q(v)=\left(v_{1}, v_{1 x}, v_{1 y}, v_{2}, v_{2 x}, v_{2 y}, v_{3}, v_{3 x}, v_{3 y}, v_{4}, v_{4 x}, v_{4 y}\right)^{\prime},
$$

where $v_{i}, v_{i x}, v_{i y}$ are the function values of $v$ and its first derivatives at vertices $a_{i}$, respectively.

Then, approximating $D(v)$ by a linear combination of the nodal parameters $Q(v)$ as follows:

$$
d_{i}(v)=v_{i},(i=1,2,3,4),
$$

for $d_{i}(v)(i=5,6,7,8)$, the numerical integrating values of cubic Hermite interpolation polynomials on the corresponding sides result in:

$$
\left\{\begin{array}{l}
d_{5}(v)=v_{1}+v_{2}+\frac{h_{x}}{3}\left(v_{1 x}-v_{2 x}\right)+O\left(h^{4}|v|_{4, K, \infty}\right), \\
d_{6}(v)=v_{2}+v_{3}+\frac{h_{y}}{3}\left(v_{2 y}-v_{3 y}\right)+O\left(h^{4}|v|_{4, K, \infty}\right), \\
d_{7}(v)=v_{3}+v_{4}+\frac{h_{x}}{3}\left(v_{4 x}-v_{3 x}\right)+O\left(h^{4}|v|_{4, K, \infty}\right), \\
d_{8}(v)=v_{1}+v_{4}+\frac{h_{y}}{3}\left(v_{1 y}-v_{4 y}\right)+O\left(h^{4}|v|_{4, K, \infty}\right),
\end{array}\right.
$$

for $d_{i}(v)(i=9,10,11,12)$, the trapezoidal rule of numerical integration giving:

$$
\left\{\begin{array}{l}
d_{9}(v)=h_{y}\left(v_{1 y}+v_{2 y}\right)+O\left(h^{3}|v|_{3, K, \infty}\right), \\
d_{10}(v)=h_{x}\left(v_{2 x}+v_{3 x}\right)+O\left(h^{3}|v|_{3, K, \infty}\right), \\
d_{11}(v)=h_{y}\left(v_{3 y}+v_{4 y}\right)+O\left(h^{3}|v|_{3, K, \infty}\right), \\
d_{12}(v)=h_{x}\left(v_{1 x}+v_{4 x}\right)+O\left(h^{3}|v|_{3, K, \infty}\right) .
\end{array}\right.
$$

The above discretizations can be rewritten in matrix form as:

$$
D(v)=G Q(v)+E(v),
$$


where:

$$
G=\left(\begin{array}{cccccccccccc}
1 & 0 & 0 & 0 & 0 & 0 & 0 & 0 & 0 & 0 & 0 & 0 \\
0 & 0 & 0 & 1 & 0 & 0 & 0 & 0 & 0 & 0 & 0 & 0 \\
0 & 0 & 0 & 0 & 0 & 0 & 1 & 0 & 0 & 0 & 0 & 0 \\
0 & 0 & 0 & 0 & 0 & 0 & 0 & 0 & 0 & 1 & 0 & 0 \\
1 & \frac{h_{x}}{3} & 0 & 1 & -\frac{h_{x}}{3} & 0 & 0 & 0 & 0 & 0 & 0 & 0 \\
0 & 0 & 0 & 1 & 0 & \frac{h_{y}}{3} & 1 & 0 & -\frac{h_{y}}{3} & 0 & 0 & 0 \\
0 & 0 & 0 & 0 & 0 & 0 & 1 & -\frac{h_{x}}{3} & 0 & 1 & \frac{h_{x}}{3} & 0 \\
1 & 0 & \frac{h_{y}}{3} & 0 & 0 & 0 & 0 & 0 & 0 & 1 & 0 & -\frac{h_{y}}{3} \\
0 & 0 & h_{y} & 0 & 0 & h_{y} & 0 & 0 & 0 & 0 & 0 & 0 \\
0 & 0 & 0 & 0 & h_{x} & 0 & 0 & h_{x} & 0 & 0 & 0 & 0 \\
0 & 0 & 0 & 0 & 0 & 0 & 0 & 0 & h_{y} & 0 & 0 & h_{y} \\
0 & h_{x} & 0 & 0 & 0 & 0 & 0 & 0 & 0 & 0 & h_{x} & 0
\end{array}\right)
$$

and:

$$
E(v)=\left(0,0,0,0, \varepsilon_{1}(v), \varepsilon_{1}(v), \varepsilon_{1}(v), \varepsilon_{1}(v), \varepsilon_{2}(v), \varepsilon_{2}(v), \varepsilon_{2}(v), \varepsilon_{2}(v)\right)^{\prime}
$$

with $\varepsilon_{1}(v)=O\left(h^{4}|v|_{4, K, \infty}\right), \varepsilon_{2}(v)=O\left(h^{3}|v|_{3, K, \infty}\right)$ From (18), it is obvious that the term $E(v)$ does not affect the convergence properties, and we neglect it and introduce a new set of parameters:

$$
\bar{Q}(v)=\left(q_{1}(v), q_{2}(v), \cdots, q_{12}(v)\right)^{\prime}
$$

such that:

$$
D(v)=G \bar{Q}(v)
$$

where $q_{1}(v)=v\left(a_{1}\right), q_{4}(v)=v\left(a_{2}\right), q_{7}(v)=v\left(a_{3}\right), q_{10}(v)=v\left(a_{4}\right), q_{2}(v)=\bar{v}_{x}\left(a_{1}\right), q_{5}(v)=\bar{v}_{x}\left(a_{2}\right)$, $q_{8}(v)=\bar{v}_{x}\left(a_{3}\right), q_{11}(v)=\bar{v}_{x}\left(a_{4}\right), q_{3}(v)=\bar{v}_{y}\left(a_{1}\right), q_{6}(v)=\bar{v}_{y}\left(a_{2}\right), q_{9}(v)=\bar{v}_{y}\left(a_{3}\right), q_{12}(v)=\bar{v}_{y}\left(a_{4}\right)$, and $\bar{v}_{x}(a)$ and $\bar{v}_{y}(a)$ have perturbations on $v_{x}(a)$ and $v_{y}(a)$, respectively. The readers are referred to $[23,24]$ for details.

Then, using (10) and (19), we obtain:

$$
b=M^{-1} G \bar{Q}(v)
$$

which in conjunction with (9) leads to the real shape function $v$. Thus, the unknowns of the discrete system are $\bar{Q}(v)$, and the real shape function space $\bar{P}(K)$ is a subspace of $P(K)$ :

$$
\bar{P}(K)=\left\{v \in P(K) ; v=\left(P, M^{-1} G q\right), \forall q \in \mathbb{R}^{12}\right\} \text {. }
$$

It is easy to check that $\operatorname{rank}(G)=10$, i.e., the dimension of $\bar{P}(K)$ is 10 , and $v \in \bar{P}(K)$ can be expressed as $v=\sum_{i=1}^{12} \beta_{i} p_{i}$, where:

$$
\left\{\begin{array}{l}
\beta_{1}=q_{1}, \beta_{2}=q_{4}, \beta_{3}=q_{7}, \beta_{4}=q_{10} \\
\beta_{5}=\frac{h_{x}}{8}\left(q_{2}-q_{5}-q_{8}+q_{11}\right), \beta_{6}=\frac{h_{y}}{8}\left(q_{3}+q_{6}-q_{9}-q_{12}\right) \\
\beta_{7}=\frac{h_{x}}{8}\left(-q_{2}+q_{5}-q_{8}+q_{11}\right), \beta_{8}=\frac{h_{y}}{8}\left(-q_{3}+q_{6}-q_{9}+q_{12}\right) \\
\beta_{9}=\frac{1}{8}\left(-q_{1}+q_{4}+q_{7}-q_{10}\right)+\frac{h_{x}}{8}\left(-q_{2}-q_{5}-q_{8}-q_{11}\right)+\frac{h_{y}}{24}\left(-q_{3}+q_{6}-q_{9}+q_{12}\right) \\
\beta_{10}=\frac{1}{8}\left(-q_{1}-q_{4}+q_{7}+q_{10}\right)+\frac{h_{x}}{24}\left(-q_{2}+q_{5}-q_{8}+q_{11}\right)+\frac{h_{y}}{8}\left(-q_{3}-q_{6}-q_{9}-q_{12}\right) \\
\beta_{11}=0, \beta_{12}=0
\end{array}\right.
$$


Obviously, $v \in \bar{P}(K)$ is a cubic polynomial. In other words, $\bar{P}(K)$ is a completely cubic polynomial space. We denote the corresponding FE space by $V_{h}$ :

$$
V_{h}=\left\{v ;\left.v\right|_{K}=\left(P, M^{-1} G q\right), \forall q \in \mathbb{R}^{12}, K \in T_{h}, v(a)=\bar{v}_{x}(a)=\bar{v}_{y}(a)=0 \text {, for any nodes } a \in \Gamma\right\} .
$$

Lemma 1. For any $v_{h} \in V_{h}$, the following properties hold:

(R1) $v_{h}$ is continuous at the vertices of elements and is zero at the vertices on $\Gamma$.

(R2) $\int_{F} v_{h} d s$ is continuous across the element edge $F$ and is zero on $F \subset \Gamma$.

(R3) $\forall p(s) \in P_{1}(F)$, i.e., $p(s)$ is a linear polynomial function defined on the element edge $F$, and $\int_{F} p(s) \frac{\partial v_{h}}{\partial n} d s$ is continuous across the element edge $F$ and is zero on $F \subset \Gamma$.

(R4) $\|\cdot\|_{h}$ is a norm over $V_{h}$, where $\|\cdot\|_{h}=\left(\sum_{K \in T_{h}}|\cdot|_{2, K}^{2}\right)^{\frac{1}{2}}$.

Proof. From (8) and (19), we know that $D\left(v_{h}\right)$ is continuous between elements and:

$$
\left\{\begin{array}{l}
d_{1}=q_{1}, d_{2}=q_{4}, d_{3}=q_{7}, d_{4}=q_{10} \\
d_{5}=q_{1}+q_{4}+\frac{h_{x}}{3}\left(q_{2}-q_{5}\right), d_{6}=q_{4}+q_{7}+\frac{h_{y}}{3}\left(q_{6}-q_{9}\right), \\
d_{7}=q_{7}+q_{10}+\frac{h_{x}}{3}\left(q_{11}-q_{8}\right), d_{8}=q_{1}+q_{10}+\frac{h_{y}}{3}\left(q_{3}-q_{12}\right) \\
d_{9}=h_{y}\left(q_{3}+q_{6}\right), d_{10}=h_{x}\left(q_{5}+q_{8}\right), d_{11}=h_{y}\left(q_{9}+q_{12}\right), d_{12}=h_{x}\left(q_{2}+q_{11}\right) .
\end{array}\right.
$$

Furthermore, it follows from (22) that:

$$
\begin{aligned}
& \int_{F_{1}} s \frac{\partial v_{h}}{\partial n} d s=\frac{h_{x}}{3}\left(q_{3}-q_{6}\right), \int_{F_{2}} s \frac{\partial v_{h}}{\partial n} d s=\frac{h_{y}}{3}\left(q_{8}-q_{5}\right), \\
& \int_{F_{3}} s \frac{\partial v_{h}}{\partial n} d s=\frac{h_{x}}{3}\left(q_{9}-q_{12}\right), \int_{F_{4}} s \frac{\partial v_{h}}{\partial n} d s=\frac{h_{y}}{3}\left(q_{2}-q_{11}\right),
\end{aligned}
$$

which together with (24) and the definition of $d_{i}(i=1,2, \ldots, 12)$ yields $(R 1),(R 2)$, and $(R 3)$.

At the same time, suppose $\left\|v_{h}\right\|_{h}=0$, then for any $K \in T_{h}$, it holds that $\left.v_{h}\right|_{K} \in P_{1}(K)$. From $(R 1)$ and $(R 2)$, we can immediately get $v_{h} \equiv 0$; thus, $(R 4)$ holds true.

\section{High Accuracy Analysis}

We consider the discrete approximation form of the variational inequality (3) as:

$$
\left\{\begin{array}{l}
\text { Find } u_{h} \in V_{h} \text {, such that } \forall v_{h} \in V_{h} \\
a\left(u_{h}, v_{h}-u_{h}\right)+\sum_{K \in T_{h}} \int_{K} \frac{\kappa}{2}\left[\left(v_{h}-\psi\right)_{-}\right]^{2} d x d y-\sum_{K \in T_{h}} \int_{K} \frac{\kappa}{2}\left[\left(u_{h}-\psi\right)_{-}\right]^{2} d x d y \geq\left(f, v_{h}-u_{h}\right),
\end{array}\right.
$$

where $a_{h}\left(w_{h}, v_{h}\right)=\sum_{K \in T_{h}} \int_{K}\left[\triangle w_{h} \triangle v_{h}+(1-v)\left(2 w_{h x y} v_{h x y}-w_{h x x} v_{h y y}-w_{h y y} v_{h x x}\right)\right] d x d y$.

It was shown in [1] that the problem (26) is equivalent to the discrete approximation of the plate problem:

$$
\left\{\begin{array}{l}
\text { Find } u_{h} \in V_{h}, \text { such that } \forall v_{h} \in V_{h} \\
a_{h}\left(u_{h}, v_{h}\right)+\sum_{K \in T_{h}} \int_{K} \kappa\left(u_{h}-\psi\right)_{-} v_{h} d x d y=\left(f, v_{h}\right) .
\end{array}\right.
$$

which is also equivalent to the minimization problem:

$$
\text { Find } u_{h} \in V_{h} \text {, such that } \forall v_{h} \in V_{h} J_{h}\left(u_{h}\right) \leq J_{h}\left(v_{h}\right)
$$

where the energy functional $J_{h}\left(v_{h}\right)=\frac{1}{2} a_{h}\left(v_{h}, v_{h}\right)+\frac{1}{2} \sum_{K \in T_{h}} \int_{K} \kappa\left[\left(v_{h}-\psi\right)_{-}\right]^{2} d x d y-\left(f, v_{h}\right)$. Since $a_{h}(\cdot, \cdot)$ is coercive on $V_{h}$, we can get the lower bound for $J_{h}\left(v_{h}\right)$ and $J_{h}\left(v_{h}\right) \rightarrow \infty$ (as $\left\|v_{h}\right\|_{h} \rightarrow \infty$ ). Then, using the elementary inequality $\left(t_{-}-s_{-}\right)(t-s) \geq 0$ and an argument similar to that used in [10], we have: 
Theorem 1. The problem (26) has a unique solution $u_{h}$. Moreover, $\left\|u_{h}\right\|_{h}$ and $\left\|\left(u_{h}-\psi\right)_{-}\right\|_{0}$ are uniformly bounded independently of $h$.

In what follows, we will give error estimates for (26).

Theorem 2. Assume that $u$ and $u_{h}$ are the solutions of (3) and (26), respectively, and the largest internal angle of $\Omega$ is $\omega<126.283696 \ldots{ }^{\circ}$. Then, we have:

$$
\left\|u-u_{h}\right\|_{h} \leq C h^{2}\left(\|u\|_{4}+\|f\|_{0}+\left\|(u-\psi)_{-}\right\|_{0}\right) .
$$

Here and later, positive constant $C$ is independent of $h$ and may be different at each appearance.

Proof. Employing the triangle inequality yields:

$$
\left\|u-u_{h}\right\|_{h} \leq\left\|u-\Pi_{h} u\right\|_{h}+\left\|\Pi_{h} u-u_{h}\right\|_{h}
$$

where $\Pi_{h}$ is the associated interpolation operator on $V_{h}$. Let $w_{h}=\Pi_{h} u-u_{h}$. We have by (27) that:

$$
\begin{aligned}
\left\|w_{h}\right\|_{h}^{2} & \leq C a_{h}\left(w_{h}, w_{h}\right)=C\left[a_{h}\left(\Pi_{h} u-u, w_{h}\right)+a_{h}\left(u, w_{h}\right)-a_{h}\left(u_{h}, w_{h}\right)\right] \\
& \leq C\left\|\Pi_{h} u-u\right\|_{h}\left\|w_{h}\right\|_{h}+C\left[a_{h}\left(u, w_{h}\right)+\sum_{K \in T_{h}} \int_{K} \kappa\left(u_{h}-\psi\right)_{-} w_{h} d x d y-\left(f, w_{h}\right)\right] .
\end{aligned}
$$

Now, we focus on the estimate of the second term on the right-hand side of (31). Obviously,

$$
\begin{aligned}
a_{h}\left(u, w_{h}\right)-\left(f, w_{h}\right)+\sum_{K \in T_{h}} \int_{K} \kappa\left(u_{h}-\psi\right)_{-} w_{h} d x d y \\
=\sum_{K \in T_{h}} \int_{\partial K} M(u) \frac{\partial w_{h}}{\partial \mathbf{n}} d s+\epsilon \sum_{K \in T_{h}} \int_{\partial K} \frac{\partial^{2} u}{\partial \mathbf{n} \partial \mathbf{s}} \frac{\partial w_{h}}{\partial \mathbf{s}} d s \\
\quad-\sum_{K \in T_{h}} \int_{K} \nabla(\triangle u) \cdot \nabla w_{h} d x d y-\left(f, w_{h}\right)+\sum_{K \in T_{h}} \int_{K} \kappa\left(u_{h}-\psi\right)_{-} w_{h} d x d y,
\end{aligned}
$$

where $M(u)=\triangle u-(1-v) \frac{\partial^{2} u}{\partial s^{2}}$ and $\epsilon=(1-v)$. In order to estimate (32), we notice that the solution $u$ of the problem (3) satisfies (see [10]):

$$
-\int_{\Omega} \nabla \Delta u \cdot \nabla v d x d y+\int_{\Omega} \kappa(u-\psi)_{-} v d x d y=(f, v), \quad \forall v \in H_{0}^{1}(\Omega) .
$$

However, from the construction of the element and (R1)-(R3) in Lemma 1, we know that $w_{h} \nsubseteq H^{1}(\Omega)$. In this situation, we need to introduce a piecewise interpolation polynomial of $w_{h}$ as follows:

$$
\left.w_{h}^{I}\right|_{K} \in P_{2}(K) \cup\left\{x^{2} y, x y^{2}\right\},\left.w_{h}^{I}\right|_{K}\left(a_{i}\right)=w_{h}\left(a_{i}\right), \int_{F} w_{h}^{I} d s=\int_{F} w_{h} d s, \forall F \subset \partial K .
$$


Then, from $(R 1)$ and $(R 2)$ in Lemma 1 , we have $w_{h}^{I} \in H_{0}^{1}(\Omega)$, which in conjunction with (32) and (33) yields:

$$
\begin{aligned}
& a_{h}\left(u, w_{h}\right)-\left(f, w_{h}\right)+\sum_{K \in T_{h}} \int_{K} \kappa\left(u_{h}-\psi\right)_{-} w_{h} d x d y \\
= & \sum_{K \in T_{h}} \int_{\partial K} M(u) \frac{\partial w_{h}}{\partial \mathbf{n}} d s+\epsilon \sum_{K \in T_{h}} \int_{\partial K} \frac{\partial^{2} u}{\partial \mathbf{n} \partial \mathbf{s}} \frac{\partial\left(w_{h}-w_{h}^{I}\right)}{\partial \mathbf{s}} d s-\sum_{K \in T_{h}} \int_{K} \nabla(\Delta u) \cdot \nabla\left(w_{h}-w w_{h}^{I}\right) d x d y \\
& -\sum_{K \in T_{h}} \int_{K} \nabla(\Delta u) \cdot \nabla w_{h}^{I} d x d y-\left(f, w_{h}\right)+\sum_{K \in T_{h}} \int_{K} \kappa\left(u_{h}-\psi\right)_{-} w_{h} d x d y \\
= & \sum_{K \in T_{h}} \int_{\partial K} M(u) \frac{\partial w_{h}}{\partial \mathbf{n}} d s+\epsilon \sum_{K \in T_{h}} \int_{\partial K} \frac{\partial^{2} u}{\partial \mathbf{n} \partial \mathbf{s}} \frac{\partial\left(w_{h}-w_{h}^{I}\right)}{\partial \mathbf{s}} d s-\sum_{K \in T_{h}} \int_{K} \nabla(\Delta u) \cdot \nabla\left(w_{h}-w_{h}^{I}\right) d x d y \\
& +\left(f, w_{h}^{I}-w_{h}\right)-\int_{\Omega} \kappa(u-\psi)_{-} w_{h}^{I} d x d y+\sum_{K \in T_{h}} \int_{K} \kappa\left(u_{h}-\psi\right)_{-} w_{h} d x d y \\
= & \sum_{K \in T_{h}} \int_{\partial K} M(u) \frac{\partial w_{h}}{\partial \mathbf{n}} d s+\epsilon \sum_{K \in T_{h}} \int_{\partial K} \frac{\partial^{2} u}{\partial \mathbf{n} \partial \mathbf{s}} \frac{\partial\left(w_{h}-w_{h}^{I}\right)}{\partial \mathbf{s}} d s-\sum_{K \in T_{h}} \int_{K} \nabla(\Delta u) \cdot \nabla\left(w_{h}-w_{h}^{I}\right) d x d y \\
& +\left(f, w_{h}^{I}-w_{h}\right)-\sum_{K \in T_{h}} \int_{K} \kappa(u-\psi)_{-}\left(w_{h}^{I}-w_{h}\right) d x d y+\sum_{K \in T_{h}} \int_{K} \kappa\left[\left(u_{h}-\psi\right)_{-}-(u-\psi)_{-}\right] w_{h} d x d y \\
\triangleq & \sum_{j=1}^{6}(E r)_{j} .
\end{aligned}
$$

Now, we start to estimate $(E r)_{j}$ one by one for $j=1,2, \ldots, 6$.

Firstly, for $\phi \in C^{0}(\Omega)$, let $P_{1}^{F} \phi$ be the piecewise linear Lagrange interpolation on edge $F$ and $P_{0}^{F} \frac{\partial w_{h}}{\partial \mathbf{n}}=\frac{1}{|F|} \int_{F} \frac{\partial w_{h}}{\partial \mathbf{n}} d s$ with $|F|=\int_{F} 1 d s$, then the corresponding remainders are:

$$
R_{1}^{F} \phi=\phi-P_{1}^{F} \phi, \quad R_{0}^{F} \frac{\partial w_{h}}{\partial \mathbf{n}}=\frac{\partial w_{h}}{\partial \mathbf{n}}-P_{0}^{F} \frac{\partial w_{h}}{\partial \mathbf{n}} .
$$

Applying (R3) in Lemma 1 leads to:

$$
\begin{gathered}
\sum_{K \in T_{h}} \sum_{F \subset \partial K} \int_{F}\left(P_{1}^{F} M(u)\right) \frac{\partial w_{h}}{\partial \mathbf{n}} d s=0, \\
\sum_{K \in T_{h}} \sum_{F \subset \partial K} \int_{F}\left(P_{1}^{F} M(u)\right)\left(P_{0}^{F} \frac{\partial w_{h}}{\partial \mathbf{n}}\right) d s=0, \sum_{K \in T_{h}} \sum_{F \subset \partial K} \int_{F} M(u)\left(P_{0}^{F} \frac{\partial w_{h}}{\partial \mathbf{n}}\right) d s=0,
\end{gathered}
$$

which follows from:

$$
\begin{aligned}
\left|(E r)_{1}\right| & =\left|\sum_{K \in T_{h}} \sum_{F \subset \partial K} \int_{F}\left(R_{1}^{F} M(u)\right)\left(R_{0}^{F} \frac{\partial w_{h}}{\partial \mathbf{n}}\right) d s\right| \\
& \leq\left|\sum_{K \in T_{h}} \sum_{F \subset \partial K}\left(\int_{F}\left(R_{1}^{F} M(u)\right)^{2} d s\right)^{\frac{1}{2}}\left(\int_{F}\left(R_{0}^{F} \frac{\partial w_{h}}{\partial \mathbf{n}}\right)^{2} d s\right)^{\frac{1}{2}}\right| \leq C h^{2}\|u\|_{4}\left\|w_{h}\right\|_{h} .
\end{aligned}
$$

Here, the Cauchy-Schwarz inequality and interpolation theorem [32,33] are used.

Secondly, for $K \in T_{h}$, we have:

$$
\begin{aligned}
& \sum_{F \subset \partial K} \int_{F}\left(P_{1}^{F} \frac{\partial^{2} u}{\partial \mathbf{n} \partial \mathbf{s}}\right) \frac{\partial\left(w_{h}-w_{h}^{I}\right)}{\partial \mathbf{s}} d s \\
& =\int_{F_{1}}\left(P_{1}^{F_{1}} \frac{\partial^{2} u}{\partial \mathbf{n} \partial \mathbf{s}}\right)\left(x, y_{K}-h_{y}\right) \frac{\partial\left(w_{h}-w_{h}^{I}\right)}{\partial x} d x-\int_{F_{3}}\left(P_{1}^{F_{3}} \frac{\partial^{2} u}{\partial \mathbf{n} \partial \mathbf{s}}\right)\left(x, y_{K}+h_{y}\right) \frac{\partial\left(w_{h}-w_{h}^{I}\right)}{\partial x} d x \\
& +\int_{F_{2}}\left(P_{1}^{F_{2}} \frac{\partial^{2} u}{\partial \mathbf{n} \partial \mathbf{s}}\right)\left(x_{K}+h_{x}, y\right) \frac{\partial\left(w_{h}-w_{h}^{I}\right)}{\partial y} d y-\int_{F_{4}}\left(P_{1}^{F_{4}} \frac{\partial^{2} u}{\partial \mathbf{n} \partial \mathbf{s}}\right)\left(x_{K}-h_{x}, y\right) \frac{\partial\left(w_{h}-w_{h}^{I}\right)}{\partial y} d y .
\end{aligned}
$$


Employing integration by parts and (34), the first term of (37) can be estimated as:

$$
\begin{aligned}
& \int_{F_{1}}\left(P_{1}^{F_{1}} \frac{\partial^{2} u}{\partial \mathbf{n} \partial \mathbf{s}}\right)\left(x, y_{K}-h_{y}\right) \frac{\partial\left(w_{h}-w_{h}^{I}\right)}{\partial x} d x \\
& =\left.\left(P_{1}^{F_{1}} \frac{\partial^{2} u}{\partial \mathbf{n} \partial \mathbf{s}}\right)\left(w_{h}-w_{h}^{I}\right)\right|_{a_{2}} ^{a_{1}}-\int_{F_{1}} \frac{d\left(P_{1}^{F_{1}} \frac{\partial^{2} u}{\partial \mathbf{n} \partial \mathbf{s}}\right)\left(x, y_{K}-h_{y}\right)}{d x}\left(w_{h}-w_{h}^{I}\right) d x=0 .
\end{aligned}
$$

Similarly, it holds that:

$$
\sum_{F \subset \partial K} \int_{F}\left(P_{1}^{F} \frac{\partial^{2} u}{\partial \mathbf{n} \partial \mathbf{s}}\right) \frac{\partial\left(w_{h}-w_{h}^{I}\right)}{\partial \mathbf{s}} d s=0,
$$

which together with interpolation theorem yields:

$$
\left|(E r)_{2}\right|=\left|\epsilon \sum_{K \in T_{h}} \sum_{F \subset \partial K} \int_{F}\left(\frac{\partial^{2} u}{\partial \mathbf{n} \partial \mathbf{s}}-P_{1}^{F} \frac{\partial^{2} u}{\partial \mathbf{n} \partial \mathbf{s}}\right) \frac{\partial\left(w_{h}-w_{h}^{I}\right)}{\partial \mathbf{s}} d s\right| \leq C h^{2}\|u\|_{4}\left\|w_{h}\right\|_{h} .
$$

Thirdly, it follows from the definition of $w_{h}^{I}$ in (34) that:

$$
\int_{K} \nabla\left(w_{h}-w_{h}^{I}\right) d x d y=\sum_{F \subset \partial K} \int_{F}\left(w_{h}-w_{h}^{I}\right) d s=0 .
$$

Then, let $P_{0}^{K} \nabla(\triangle u)=\frac{1}{|K|} \int_{K} \nabla(\triangle u) d x d y$ and $|K|=\int_{K} 1 d x d y$. With a similar argument as $(E r)_{1}$ and $(E r)_{2}$, we obtain:

$$
\left|(E r)_{3}\right|=\left|\sum_{K \in T_{h}} \int_{K}\left(\nabla(\triangle u)-P_{0}^{K} \nabla(\triangle u)\right) \cdot \nabla\left(w_{h}-w_{h}^{I}\right) d x d y\right| \leq C h^{2}\|u\|_{4}\left\|w_{h}\right\|_{h} .
$$

As for $(E r)_{4}$ and $(E r)_{5}$, from the Cauchy-Schwarz inequality and interpolation theorem, it holds that:

$$
\left|(E r)_{4}\right|=\left|f\left(w_{h}^{I}-w_{h}\right)\right| \leq\|f\|_{0}\left\|w_{h}-w_{h}^{I}\right\|_{0} \leq C h^{2}\|f\|_{0}\left\|w_{h}\right\|_{h}
$$

and:

$$
\begin{aligned}
\left|(E r)_{5}\right| & \leq \mathcal{\kappa}\left\|(u-\psi)_{-}\right\|_{0}\left\|w_{h}-w_{h}^{I}\right\|_{0} \\
& \leq h^{2}\left\|(u-\psi)_{-}\right\|_{0}\left\|w_{h}\right\|_{h} .
\end{aligned}
$$

Finally, employing the elementary inequality $\left(t_{-}-s_{-}\right)(t-s) \geq 0$ and Theorem 1 , we have:

$$
\begin{aligned}
(E r)_{6} & =\sum_{K \in T_{h}} \int_{K} \kappa\left[\left(u_{h}-\psi\right)_{-}-(u-\psi)_{-}\right]\left(\Pi_{h} u-u+u-u_{h}\right) d x d y \\
& \leq \sum_{K \in T_{h}} \int_{K} \kappa\left[\left(u_{h}-\psi\right)_{-}-(u-\psi)_{-}\right]\left(\Pi_{h} u-u\right) d x d y \\
& \leq C\left(\left\|\left(u_{h}-\psi\right)_{-}\right\|_{0}+\left\|(u-\psi)_{-}\right\|_{0}\right)\left\|\Pi_{h} u-u\right\|_{0} \leq C\left\|(u-\psi)_{-}\right\|_{0}\left\|\Pi_{h} u-u\right\|_{0} .
\end{aligned}
$$

Combining (31), (35), and the above bounds of $(E r)_{1}-(E r)_{6}$ results in:

$$
\begin{aligned}
\left\|\Pi_{h} u-u_{h}\right\|_{h}^{2} & \leq C h^{4}\left(\|u\|_{4}^{2}+\|f\|_{0}^{2}+\left\|(u-\psi)_{-}\right\|_{0}^{2}\right) \\
& +C\left(\left\|\Pi_{h} u-u\right\|_{h}^{2}+\left\|(u-\psi)_{-}\right\|_{0}\left\|\Pi_{h} u-u\right\|_{0}\right) .
\end{aligned}
$$

Therefore, the desired result (29) follows from (30), (46) and the interpolation theorem immediately.

Remark 1. $w_{h}^{I}$ plays a key role in the estimations of $(E r)_{2}$ and $(E r)_{3}$ in Theorem 2. If using the error estimate method in [10], we can only obtain the convergence result with the order of $O(h)$. In addition, it should be pointed out that the analysis presented herein is also valid for the elastic obstacle problem with $j(v)=\int_{\Omega} \kappa\left[(v-\psi)_{+}\right]^{2} d x d y, t_{+}=\max \{t, 0\}$, and $f \in L^{2}(\Omega), f \geq 0$ a.e. in $\Omega$. 
Remark 2. Compared with the work in [10], we obtain the same high accuracy result $O\left(h^{2}\right)$ with a non- $C^{0}$ nonconforming element instead of the $C^{0}$ nonconforming $A C M^{\prime}$ 's rectangular element. Therefore, our work is an extension of [10], and the requirement for the nonconforming plate element's smoothness from $C^{0}$ continuity to mean $C^{1}$ continuity is further reduced $((R 1)-(R 3)$ implies that the element discussed in this paper is of the mean $C^{1}$ type). It can be checked that the high accuracy result in Theorem 2 is no longer true for many classical non- $C^{0}$ nonconforming plate elements, such as the Morley triangle element, the Veubeke triangle element, and so on.

\section{Numerical Experiment}

In this section, we consider the elastic obstacle problem (2) with $\Omega=[0,1]^{2}, f=-10, v=0.25$, and an elastic obstacle defined by the function $\psi=\left\{\begin{array}{ll}0 & \text { if }(x, y) \in[0.3 ; 0.7]^{2}, \\ -1 & \text { otherwise. }\end{array}\right.$ Since the exact solution is unknown, we refer the numerical solution on rectangles with $h=1 / 128$ as the exact solution to show the errors. The domain $\Omega$ is divided into $N \times N$ rectangles, and the cases $\kappa=10^{j}, j=0,1,3,4$ with $N=8,16,32,64$ are computed, respectively. The numerical results are given in the following Table 1. Moreover, Figures 1-3 illustrate the numerical solution $u_{h}$ with $N=64$, and Figure 4 presents the errors in the logarithm scales. It is obvious that the errors in the energy norm are convergent at order $O\left(h^{2}\right)$, which coincides with the theoretical analysis in Theorem 2.

Table 1. Errors of $\left\|u-u_{h}\right\|_{h}$ and the CPU time.

\begin{tabular}{lccccc}
\hline$\kappa \backslash N \times N$ & $\mathbf{4} \times \mathbf{4}$ & $\mathbf{8} \times \mathbf{8}$ & $\mathbf{1 6} \times \mathbf{1 6}$ & $\mathbf{3 2} \times \mathbf{3 2}$ & $\mathbf{6 4} \times \mathbf{6 4}$ \\
\hline$\kappa=10^{0}$ & 13.50099036072611 & 3.60334964063092 & 0.88702928335453 & 0.20887990183367 & 0.04536489180482 \\
\hline time $(s)$ & 0.048482 & 0.073726 & 0.207029 & 0.667169 & 51.440274 \\
\hline$\kappa=10^{1}$ & 13.48345125601387 & 3.59535482262866 & 0.88451602203801 & 0.20828947880233 & 0.04523833745855 \\
\hline time $(s)$ & 0.108480 & 0.394770 & 2.421881 & 10.936272 & 50.397260 \\
\hline$\kappa=10^{3}$ & 11.44582259361440 & 2.88258835869155 & 0.69885436205702 & 0.16610400089376 & 0.03616945545106 \\
\hline time $(s)$ & 5.307547 & 28.444150 & 187.683438 & 793.338376 & 3411.901540 \\
\hline$\kappa=10^{4}$ & 11.241262037642660 & 2.87323216390585 & 0.62499932334938 & 0.15026200240187 & 0.03545375844482 \\
\hline time $(s)$ & 17.876946 & 99.593113 & 919.972509 & 5191.457737 & $19,710.033405$ \\
\hline
\end{tabular}

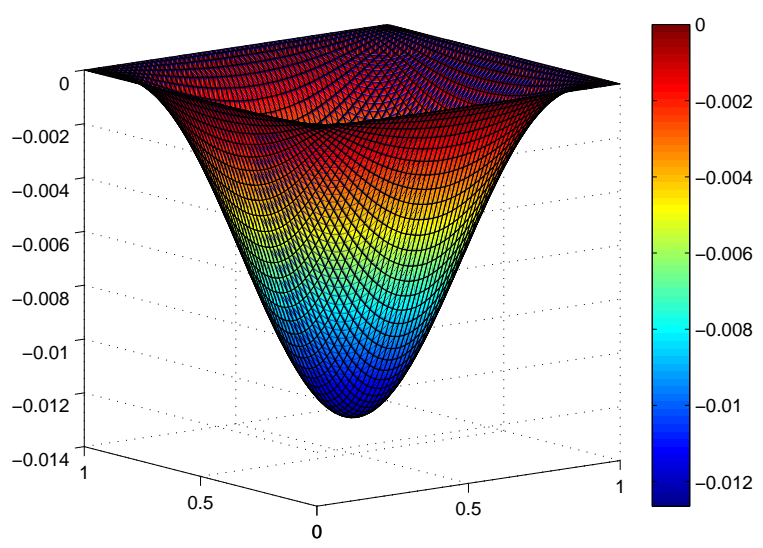

Figure 1. The numerical solution $u_{h}$ with $\kappa=10$. 


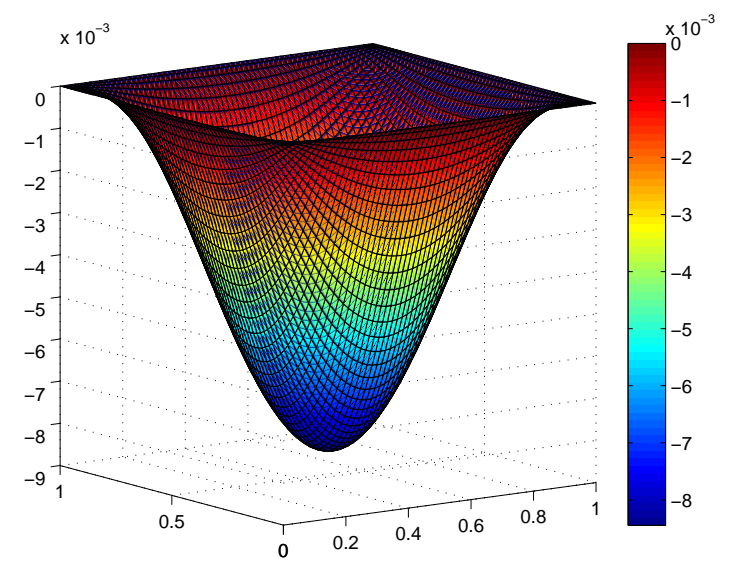

Figure 2. The numerical solution $u_{h}$ with $\kappa=10^{3}$.

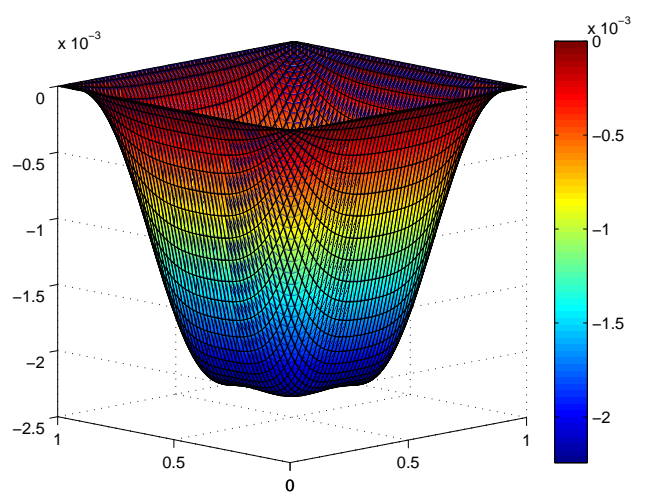

Figure 3. The numerical solution $u_{h}$ with $\kappa=10^{4}$.

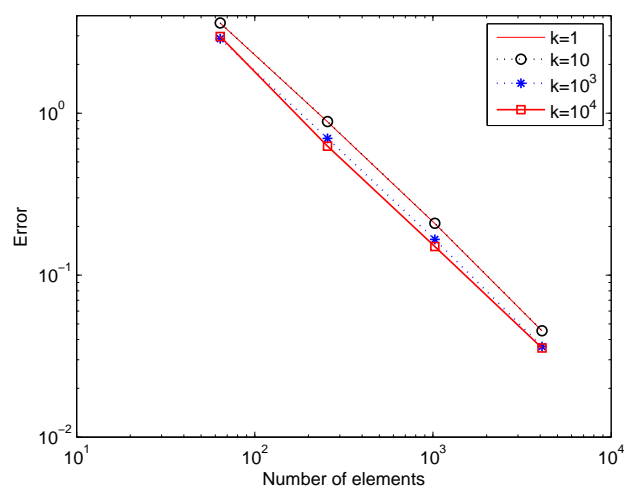

Figure 4. Errors in the energy norm with different $k$.

Author Contributions: Conceptualization, L.P.; software, L.P.; methodology, L.P. and D.S.; writing, original draft preparation, L.P.; writing, review and editing, D.S. All authors read and agreed to the published version of the manuscript.

Funding: This research was supported by the National Natural Science Foundation of China (No. 11701523, No. 11671369).

Conflicts of Interest: The authors declare no conflicts of interest. 


\section{References}

1. Tosone C.; Maceri, A. The clamped plate with elastic unilateral obstacles: A finite element approach. Math. Model. Methods Appl. Sci. 2003, 13, 1231-1243. [CrossRef]

2. Glowinski, R. Numerical Methods for Nonlinear Variational Inequality Problems; Springer: New York, NY, USA, 1984.

3. Wang, L.H. Some strongly discontinuous nonconforming finite element approximations of a fourth order variational inequality with displacement obstacle. Acta. Numer. Math. 1992, 14, 98-101.

4. Shi, D.Y.; Chen, S.C. Quasi conforming element approximation for a fourth order variational inequality with displacement obstacle. Acta Math. Sci. 2003, 23B, 61-66. [CrossRef]

5. Shi, D.Y.; Chen, S.C. General estimates on nonconforming elements for a fourth order variational problem. Numer. Math. Sin. 2003, 25, 99-106.

6. Brenner, S.C.; Sung, L.Y.; Zhang, Y. Finite element methods for the displacement obstacle problem of clamped plates. Math. Comp. 2012, 81, 1247-1262. [CrossRef]

7. Brenner, S.C.; Sung, L.Y.; Zhang, H.C.; Zhang, Y. A Morley finite element method for the displacement obstacle problem of clamped Kirchhoff plates. J. Comput. Appl. Math. 2013, 254, 31-42. [CrossRef]

8. Shi, D.Y.; Pei, L.F. Double set parameter finite element method for two-sided displacement obstacle problem of clamped plate. J. Math. Anal. Appl. 2016, 436, 203-216. [CrossRef]

9. Shi, D.Y.; Pei, L.F. A new error analysis of Bergan's energy-orthogonal element for two-sided displacement obstacle problem of clamped plate. J. Math. Anal. Appl. 2016, 442, 339-352. [CrossRef]

10. Han, W.M.; Hua, D.Y.; Wang, L.H. Nonconforming finite element methods for a clamped plate with elastic unilateral obstacle. J. Integral Eq. Appl. 2006, 18, 267-284. [CrossRef]

11. Gustafsson, T.; Stenberg, R.; Videman, J. A stabilised finite element method for the plate obstacle problem. Br. Numer. Math. 2018, 2018, 1-28. [CrossRef]

12. Li, M.X.; Lin, Q.; Zhang, S.H. Superconvergence of finite element method for the Signorini problem. J. Comput. Appl. Math. 2008, 222, 284-292. [CrossRef]

13. Shi, D.Y.; Ren, J.C.; Gong, W. Convergence and superconvergence analysis of a nonconforming finite element method for solving the Signorini problem. Nonlinear Anal. Theor. 2012, 75, 3493-3502. [CrossRef]

14. Shi, D.Y.; Guan, H.B.; Guan, X.F. Superconvergence analysis of finite element method for a second-type variational inequality. J. Appl. Math. 2012, 2012,1-12. [CrossRef]

15. Xu, C.; Shi, D.Y. Superconvergence analysis of low order nonconforming finite element methods for variational inequality problem with displacement obstacle. Appl. Math. Comp. 2019, 348,1-11. [CrossRef]

16. Chen, S.C.; Yin, L.; Mao, S.P. An anisotropic, superconvergent nonconforming plate finite element. J. Comput. Appl. Math. 2008, 220, 96-110. [CrossRef]

17. Shi, D.Y.; Wu, Y.M. Uniform superconvergent analysis of a new mixed finite element method for nonlinear Bi-wave singular perturbation problem. Appl. Math. Lett. 2019, 93, 131-138. [CrossRef]

18. Hu, J.; Shi, Z.C.; Yang, X.Q. Superconvergence of both two and three dimensional rectangular Morley elements for biharmonic equations. Mathematics 2015, 132, 491-509.

19. Ruggieri, M.; Speciale, M.P. Approximate analysis of a nonlinear dissipative model. Acta Appl. Math. 2014 , 132, 549-559. [CrossRef]

20. Shi, D.Y.; Mao, S.P.; Chen, S.C. On the anisotropic accuracy analysis of ACM's nonconforming finite element. J. Comput. Math. 2005, 23, 185-198.

21. Frehse, J. On the regularity of the solution of the biharmonic variational inequality. Manuscripta Math. 1973, 9, 91-103. [CrossRef]

22. Schild, B. A regularity result for polyharmonic variational inequalities with thin obstacles. Ann. Scuola Norm. Sup. Pisa Cl. Sci. 1984, 11, 87-122.

23. Chen, S.C.; Shi, Z.C. Double set parameter method of constructing stiffness matrices. Numer. Math. Sin. 1991, 3, 286-296.

24. Chen, S.C.; Shi, D.Y. The error estimates for double set parameter elemet. J. Eng. Math. 2005, 22, 599-605.

25. Stummel, F. The generalized patch test. SIAM J. Numer. Anal. 1979, 16, 449-471. [CrossRef]

26. Shi, Z.C. The F-E-M-test for convergence of nonconforming finite element. Math. Comp. 1987, 49, 391-405.

27. Shi, D.Y. Studies on Nonconforming Elements. Ph.D. Thesis, Xi'an Jiaotong University, Xi'an, China, 1997. 
28. Mao, S.P.; Chen, S.C. A quadrilateral nonconforming finite element for linear elasticity problem. Adv. Comput. Math. 2008, 28, 81-100. [CrossRef]

29. Chen, S.C.; Zhao, Y.C.; Shi, D.Y. Non $C^{0}$ nonconforming elements for elliptic fourth order singular perpurbation problem. J. Comput. Math. 2005, 23, 185-198.

30. Xie, P.L.; Shi, D.Y.; Li, H. A new robust $\mathrm{C}^{0}$-type nonconforming triangular element for singular perturbation problems. Appl. Math. Comput. 2010, 217, 3832-3843. [CrossRef]

31. Chen, S.C.; Liu, M.F.; Qiao, Z.H. An anisotropic nonconforming element for fourth order elliptic singular perturbation problem. Int. J. Numer. Anal. and Model. 2010, 7, 766-784.

32. Ciarlet, P.G. The Finite Element Method for Elliptic Problems; North-Holland: Amsterdam, The Netherlands, 1978.

33. Brenner, S.C.; Scott, L.R. Mathematics Theory Finite Element Methods; Springer: New York, NY, USA, 1994.

Publisher's Note: MDPI stays neutral with regard to jurisdictional claims in published maps and institutional affiliations.

(C) 2020 by the authors. Licensee MDPI, Basel, Switzerland. This article is an open access article distributed under the terms and conditions of the Creative Commons Attribution (CC BY) license (http://creativecommons.org/licenses/by/4.0/). 